\title{
MRI Features May Predict Molecular Features of Glioblastoma in Isocitrate Dehydrogenase Wild-Type Lower- Grade Gliomas
}

\author{
(D) C.J. Park, (D) K. Han, (D) H. Kim, (DS.S. Ahn, (DD. Choi, (D).W. Park, DJ.H. Chang, (DS.H. Kim, (DS. Cha, and (DS.-K. Lee
} on $=$

\begin{abstract}
BACKGROUND AND PURPOSE: Isocitrate dehydrogenase (IDH) wild-type lower-grade gliomas (histologic grades II and III) with epidermal growth factor receptor (EGFR) amplification or telomerase reverse transcriptase (TERT) promoter mutation are reported to behave similar to glioblastoma. We aimed to evaluate whether MR imaging features could identify a subset of IDH wild-type lower-grade gliomas that carry molecular features of glioblastoma.
\end{abstract}

MATERIALS AND METHODS: In this multi-institutional retrospective study, pathologically confirmed IDH wild-type lower-grade gliomas from 2 tertiary institutions and The Cancer Genome Atlas constituted the training set (institution 1 and The Cancer Genome Atlas, 64 patients) and the independent test set (institution 2, 57 patients). Preoperative MRIs were analyzed using the Visually AcceSAble Rembrandt Images and radiomics. The molecular glioblastoma status was determined on the basis of the presence of EGFR amplification and TERT promoter mutation. Molecular glioblastoma was present in $73.4 \%$ and $56.1 \%$ in the training and test sets, respectively. Models using clinical, Visually AcceSAble Rembrandt Images, and radiomic features were built to predict the molecular glioblastoma status in the training set; then they were validated in the test set.

RESULTS: In the test set, a model using both Visually AcceSAble Rembrandt Images and radiomic features showed superior predictive performance (area under the curve $=0.854$ ) than that with only clinical features or Visually AcceSAble Rembrandt Images (areas under the curve $=0.514$ and 0.648 , respectively; $P<.001$, both). When both Visually AcceSAble Rembrandt Images and radiomics were added to clinical features, the predictive performance significantly increased (areas under the curve $=0.514$ versus 0.863 , $P<.001)$.

CONCLUSIONS: MR imaging features integrated with machine learning classifiers may predict a subset of IDH wild-type lowergrade gliomas that carry molecular features of glioblastoma.

ABBREVIATIONS: $\mathrm{AUC}=$ area under the receiver operating characteristic curve; cIMPACT-NOW = Consortium to Inform Molecular and Practical Approaches to CNS Tumor Taxonomy; GBM = glioblastoma; LASSO = least absolute shrinkage and selection operator; RFE = recursive feature elimination; SVM = support vector machine; TCGA = The Cancer Genome Atlas; VASARI = Visually AcceSAble Rembrandt Images; WHO = World Health Organization

A mutation in the isocitrate dehydrogenase $(I D H)$ gene is a major classifier that leads to the stratification of gliomas with significantly different survival rates among the lower-grade gliomas (World Health Organization [WHO] grades II and III) as well as glioblastomas (GBMs). ${ }^{1-4} I D H$ wild-type tumors, which

Received June 15, 2020; accepted after revision October 19.

From the Department of Radiology (C.J.P.), Yonsei University College of Medicine, Yongin-si, Gyeonggi-do, Korea; Department of Radiology (K.H., H.K.,

S.S.A., Y.W.P., S.-K.L.), Research Institute of Radiological Sciences, Center for Clinical Imaging Data Science, Department of Neurosurgery (J.H.C.), and Department of Pathology (S.H.K.), Yonsei University College of Medicine, Seoul, Korea; Department of Computer Science (D.C.), Yonsei University, Seoul, Korea; and Department of Radiology and Biomedical Imaging (S.C.), University of California San Francisco, San Francisco, California.

This work was supported by the Basic Science Research Program through the National Research Foundation of Korea funded by the Ministry of Science, Information and Communication Technologies \& Future Planning (2017RIDIA1B03030440 and 2020RIA2C1003886). account for $<30 \%$ of the histologic grade II and III gliomas, show worse prognoses than those with the IDH mutation. ${ }^{1,5,6}$

Previous studies have reported heterogeneous clinical outcomes among the $I D H$ wild-type lower-grade gliomas according to a variable combination of genetic profiles. ${ }^{7-9}$ Recently, the Consortium to Inform Molecular and Practical Approaches to CNS Tumor Taxonomy (cIMPACT-NOW) provided a new designation for gliomas, namely, "diffuse astrocytic glioma, IDH-

Please address correspondence to Sung Soo Ahn, MD, PhD, Department of Radiology, Research Institute of Radiological Science, and Center for Clinical Image Data Science, College of Medicine, Yonsei University, 50 Yonsei-ro, Seodaemungu, Seoul 03722, Korea; e-mail: sungsoo@yuhs.ac

- Indicates open access to non-subscribers at www.ajnr.org

Indicates article with online supplemental data.

http://dx.doi.org/10.3174/ajnr.A6983 
wildtype, with molecular features of GBM, WHO grade IV," which corresponds to histologic grades II and III IDH wild-type gliomas showing high-level epidermal growth factor receptor (EGFR) amplification, the combination of a whole chromosome 7 gain and a whole chromosome 10 loss $(+7 /-10)$, or telomerase reverse transcriptase (TERT) promotor mutations. ${ }^{10}$ These specifications emphasize that the $I D H$ wild-type lower-grade gliomas that fulfill these molecular criteria will follow an aggressive clinical course closely resembling that of an IDH wild-type GBM. Therefore, it is highly desirable that MR imaging predict the tumors with specific molecular features that would have a worse prognosis than the others. Especially, this characteristic will be clinically relevant in cases in which detailed genetic profiling cannot be performed.

The Visually AcceSAble Rembrandt Images (VASARI; https:// wiki.nci.nih.gov/display/CIP/VASARI) are a standardized feature set that was developed to describe MR imaging features of gliomas using a standardized vocabulary. It provides 26 distinct imaging lexicons that allow accurate, reproducible, and comprehensive assessment of the gliomas. Previous studies have reported that the VASARI assessment was highly reproducible, clinically meaningful, and biologically relevant in glioblastomas. ${ }^{11-13}$

Radiomics extracts high-dimensional quantitative imaging features, such as intensity distributions, spatial relationships, textural heterogeneity, and shape descriptors; ${ }^{14}$ hidden information can be revealed using radiomics. ${ }^{15}$ In particular, a strength of radiomics is that it reflects intratumoral heterogeneity by a variety of mathematic methods used to quantify the gray-level spatial variations within an image to derive textural features. ${ }^{16}$ Several previous studies have applied radiomics to predict specific genetic mutations in patients with lower-grade gliomas, including EGFR expression. ${ }^{17-19}$ The predictive role of radiomics for EGFR amplification or TERT promotor mutation in patients with $I D H$ wild-type lower-grade gliomas is clinically relevant in the light of cIMPACT-NOW recommendations; however, it has not been studied to date.

Our study aimed to evaluate whether comprehensive analysis of MR imaging features using the VASARI set and radiomics can identify a subset of IDH wild-type lower-grade gliomas with molecular features that may also follow a clinical course similar to that of GBM.

\section{MATERIALS AND METHODS}

This retrospective study was approved by the institutional review boards of the 2 academic institutions, Yonsei University Health System (Seoul, Korea) (institution 1) and University of California, San Francisco Medical Center (California, United States) (institution 2). The requirement for obtaining informed patient consent was waived. For another dataset, the publicly available National Institutes of Health/National Cancer Institute-approved Cancer Genome Atlas (TCGA) and The Cancer Imaging Archive data bases in which all data are anonymized were used. ${ }^{20}$ Thus, individual institutional approval was not required for using the TCGA dataset.

\section{Patients}

Patients with pathologically confirmed lower-grade gliomas were identified in each institution and in the TCGA dataset. The inclusion criteria were as follows: 1) IDH wild-type; 2) with preoperative MR imaging; 3) with known specific molecular features of GBM. According to cIMPACT-NOW, EGFR amplification, chromosome $+7 /-10$, and TERT promotor mutation determine whether IDH wild-type lower-grade gliomas have the molecular features of GBM. However, because the chromosome +7/-10 status was not available in the test set, this feature was excluded from our analysis.

The patients were divided into 2 subsets according to their EGFR amplification and TERT promotor mutation status: If either the EGFR amplification or the TERT promotor mutation status was positive, the tumor was considered an $I D H$ wild-type lower-grade glioma with the molecular features of GBM. If both the EGFR amplification and TERT promotor mutation status were negative, the tumor was considered an IDH wild-type lower-grade glioma without the molecular features of GBM. The primary outcome was the status of molecular features of GBM. The patients' ages and WHO grades were retrieved from the electronic medical records.

\section{Training Set: Institution $1+$ TCGA}

Institution 1 and TCGA data constituted the training set. We identified 166 patients with pathologically confirmed lower-grade gliomas from January 2012 to December 2018 at the University of California, San Francisco. The exclusion criteria were as follows: 1) $I D H$-mutant tumors $(n=47)$; 2$)$ unknown $I D H$ mutation status $(n=3)$; 3$)$ history of brain surgery $(n=59) ; 4)$ younger than 18 years of age $(n=7) ; 5)$ without preoperative MR imaging $(n=3)$; and 6) unknown status of molecular features of GBM $(n=15)$. Thirty-two patients with IDH wild-type lowergrade gliomas were enrolled (Fig 1). Among the 32 patients, 21 patients (65.6\%) had IDH wild-type lower-grade gliomas with molecular features of GBM.

The TCGA (http://cancergenome.nih.gov) provided the clinical and MR imaging data of 199 lower-grade gliomas in patients older than 18 years of age. The exclusion criteria were as follows: 1) $I D H$-mutant tumors $(n=151)$; 2) unknown $I D H$ mutation status $(n=2)$; and 3) unknown status of molecular features of GBM $(n=14)$. Thirty-two patients with IDH wild-type lowergrade gliomas were enrolled (Fig 1). Among 32 patients, 26 patients (81.3\%) had IDH wild-type lower-grade gliomas with molecular features of GBM.

In the training set, 25 and 39 patients had WHO grade II and III gliomas, respectively. Among 47 patients with molecular features of GBM, 32 patients had WHO grade III gliomas (32/47, $68.1 \%)$ and 15 patients had WHO grade II gliomas (15/47, $31.9 \%)$.

\section{Test Set: Institution 2}

From January 2007 to October 2018, four hundred eighty-six patients with pathologically confirmed lower-grade gliomas were identified. The exclusion criteria were as follows: 1) IDH-mutant tumors $(n=210) ; 2)$ unknown $I D H$ mutation status $(n=85) ; 3)$ history of brain surgery $(n=10) ; 4)$ younger than 18 years of age ( $n=13)$; 5) without preoperative MR imaging $(n=11)$; and 6$)$ unknown status of molecular features of GBM $(n=100)$. Finally, 57 patients constituted an independent test set (Fig 1). Among 


\section{Institution 1}

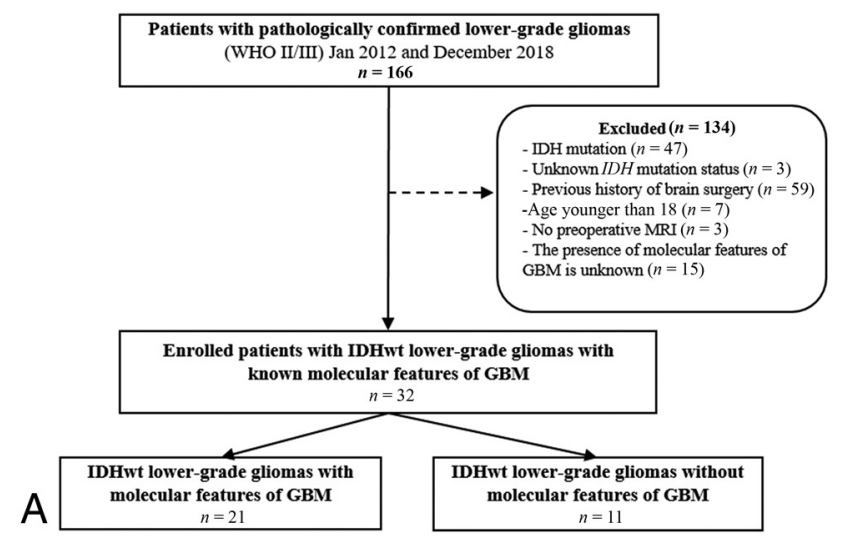

Institution 2

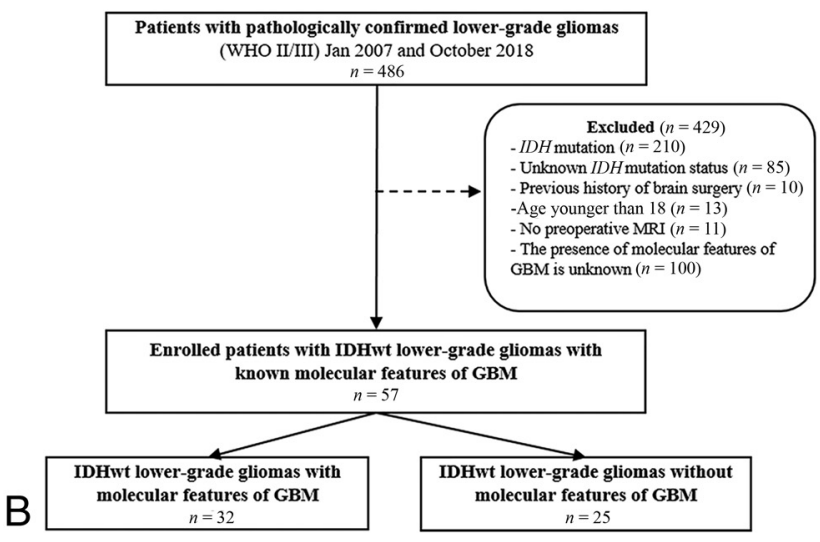

\section{TCGA}

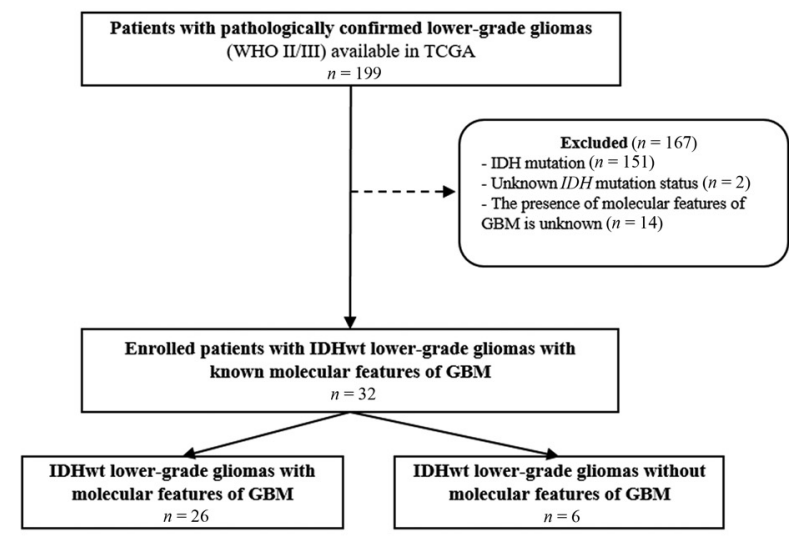

FIG 1. Flow chart showing the distribution of the patient population in the training $(A)$ and the test $(B)$ sets. IDHwt indicates isocitrate dehydrogenase wild-type.

the 57 patients, 32 patients (56.1\%) had IDH wild-type lowergrade gliomas with molecular features of GBM.

In the test set, 23 and 34 patients had WHO grade II and III gliomas, respectively. Among 32 patients with molecular features of GBM, 15 patients had WHO grade III gliomas (15/32, 46.9\%) and 17 patients had WHO grade II gliomas (17/32, 53.1\%).

\section{MR Image Acquisition}

Patients from institutions 1 and 2 both underwent brain MRI with a 3 T system (institution 1, Discovery, GE Healthcare; institution 2, Achieva or Ingenia, Philips Healthcare). The detailed parameters of MR imaging sequences from each institution are illustrated in the Online Supplement Data.

\section{Image Analysis}

Two board-certified neuroradiologists (12 and 3 years' experience, respectively) independently reviewed the MR images of all patients according to the VASARI feature set, blinded to patients' clinical information. Discrepancies between them were settled by consensus. The VASARI lexicon provides 26 imaging descriptors based on T1, T2, FLAIR, and DWI. Diffusion features were not evaluated because many patients lacked preoperative DWI in TCGA cohorts. We analyzed the following MR imaging features: location (lobar/nonlobar), side of lesion center (midline or not), eloquent brain involvement, presence of enhancement, proportion of enhancing tumor, nonenhancing tumor, edema and necrosis, presence of cysts, multifocality, expansile or infiltrative growth, margin of nonenhancing tumor (well-defined or poorly-defined), hemorrhage, pial invasion, ependymal extension, cortical involvement, deep white matter involvement, midline cross, and satellites. Detailed descriptions of all features are available at the National Cancer Institute's Cancer Imaging Archive (https://wiki.cancerimagingarchive. net/display/Public/VASARI+Research+Project) ${ }^{21}$

\section{Image Preprocessing and Radiomic Feature Extraction}

First, T2WI and postcontrast T1 images were resampled to an identical spatial resolution of $1 \times 1 \times 1 \mathrm{~mm}$ using Nilearn (https:// nilearn.github.io). These images were subjected to N4 bias correction to remove low-frequency intensity and nonuniformity. ${ }^{22,23}$ After resampling and N4 bias correction, postcontrast T1 images were registered to identical spatial coordinates using T2WI as a template with SimpleITK (http://www.simpleitk.org). Signal intensity was normalized using the WhiteStripe $\mathrm{R}$ package, ${ }^{24}$ which is implemented in $\mathrm{R}$ software (Version 3.5.1; www. R-project.org). The ROI was drawn by a neuroradiologist and 


\begin{tabular}{|c|c|c|c|c|}
\hline \multirow[b]{2}{*}{ Clinical Characteristics } & \multicolumn{2}{|c|}{ Training Set } & \multirow[b]{2}{*}{ Test Set, Institution 2} & \multirow[b]{2}{*}{$P$ Value } \\
\hline & Institution 1 & TCGA & & \\
\hline No. of patients & 32 & 32 & 57 & \\
\hline Age (yr) & $49.0[S D, 19.1]$ & $51.1[S D, 14.7]$ & $53.4[S D, 16.2]$ & .291 \\
\hline Sex (male/female) & 19:13 & $17: 15$ & $27: 30$ & .901 \\
\hline WHO grade & & & & .800 \\
\hline Grade II & $16(50.0 \%)$ & $9(28.1 \%)$ & $23(40.4 \%)$ & \\
\hline Grade III & $16(50.0 \%)$ & $23(71.9 \%)$ & $34(59.6 \%)$ & \\
\hline Molecular GBM status & & & & .053 \\
\hline With molecular features of GBM & $21(65.6 \%)$ & $26(81.3 \%)$ & $32(56.1 \%)$ & \\
\hline Without molecular features of GBM & $11(34.4 \%)$ & $6(18.7 \%)$ & $25(43.9 \%)$ & \\
\hline
\end{tabular}

confirmed by another neuroradiologist ( 3 and 12 years' experience, respectively) to segment the infiltrative tumor and edemadefined with high signal intensity on T2WI-using a semiautomatic method of signal intensity threshold with the Medical Image Processing, Analysis, and Visualization software, Version 7.0 (National Institutes of Health; mipav.cit.nih.gov). The radiomic features were extracted from the ROIs on T2WI and postcontrast T1 images using PY RADIOMICS 1.2.0 (http://www. radiomics.io/pyradiomics.html). ${ }^{25}$

Twelve shapes, 18 first-orders, 23 gray-level co-occurrence matrices, gray-level run length matrices, 16 gray-level size zone matrices, and 5 neighborhood gray tone difference matrices were extracted from the ROIs on T2WI and postcontrast T1 images, constituting a total of 180 radiomic features.

\section{Pathologic Evaluation and Molecular Subtyping}

In the 2 academic institutions (institution 1 and 2), all surgical specimens were histopathologically diagnosed according to the 2016 WHO classification. Both peptide nucleic acid-mediated clamping polymerase chain reaction and immunohistochemical analysis were performed to detect the IDH1-R132H mutation. ${ }^{1}$ Monoclonal antibody H09 was used for immunohistochemical analysis. The degree of IDH1-R132H staining was considered positive if stained cells were observed, while specimens without stained cells were deemed negative. ${ }^{26,27}$ In IDH1-negative cases, the IDH1/2 status was confirmed by the peptide nucleic acidmediated clamping polymerase chain reaction. Targeted nextgeneration sequencing was performed using the TruSight Tumor 170 panel (Illumina; https:/www.illumina.com/products/ by-type/clinical-research-products/trusight-tumor-170.html). ${ }^{28,29}$ For copy number analysis, EGFR genes with greater than a 2-fold change relative to the average level were considered to have undergone amplification. The TERT promotor mutation was determined using a pyrosequencing assay, and the C228T and C250T mutations were analyzed, as described previously. ${ }^{29}$

In the TCGA dataset, the detailed information of molecular subtyping is provided in the Genomic Data Commons Data Portal of The Cancer Genome Atlas Low Grade Glioma (TCGA-LGG) data collection (https:/www.cancer.gov/about-nci/organization/ $\mathrm{ccg} /$ research/structural-genomics/tcga/studied-cancers/glioma). ${ }^{20}$

\section{Feature Selection and Classification Methods}

Feature selection and classification methods were performed using $\mathrm{R}$ software (Version 3.5.1). To avoid collinearity and minimize the potential risk of overfitting while handling highdimensional radiomic features, ${ }^{30,31}$ we used the least absolute shrinkage and selection operator (LASSO) and recursive feature elimination (RFE) to select the important features using the caret $\mathrm{R}$ package. ${ }^{32}$ Feature selection was performed before model construction using either LASSO, RFE, or RFE + LASSO, when LASSO was performed after RFE to further minimize the redundant features. Three subsets of selected features were combined with 4 different machine learning classifiers: XGboost (https://xgboost.ai), support vector machine (SVM), linear discriminant analysis, and adaptive boosting. The performance of the feature-selection methods + classifiers was tested using 5 -fold cross-validation with 3 repetitions to enhance the generalizability of our results.

\section{Statistical Analysis}

Statistical analysis was performed using $\mathrm{R}$ software (Version 3.5.1).

Interobserver agreement for assessing VASARI features was expressed with the weighted $\kappa$ coefficients as follows: $<0.20$, poor; $0.21-0.40$, fair; $0.41-0.60$, moderate; $0.61-0.80$, good; $0.81-$ 1.00 , excellent. ${ }^{33}$

In the training set, there were 4 different models: model 1, clinical features, only age and WHO grade; model 2, VASARI features only; model 3, VASARI + radiomic features; and model 4 , clinical + VASARI + radiomic features. Multivariable logistic regression was used to develop models 1 and 2: model 1 with 2 clinical features, patient age and WHO grade; model 2 with VASARI features. In models 3 and 4 , twelve combinations of the aforementioned feature-selection methods and machine learning classifiers were used. These 4 models were validated in the test set. Receiver operating characteristic curves were obtained, and the area under the curve (AUC) was calculated to measure the predictive performance. The AUCs from different models were compared by mean of the Delong method, and multiple comparisons were corrected using the BenjaminiHochberg procedure. ${ }^{34}$

\section{RESULTS}

The characteristics of enrolled patients from all 3 datasets are summarized in Table 1 . The proportions of $I D H$ wild-type lowergrade gliomas with molecular features of GBM were 73.4\% (47/ $64)$ and $56.1 \%$ (32/57) in the training and test sets, respectively. 


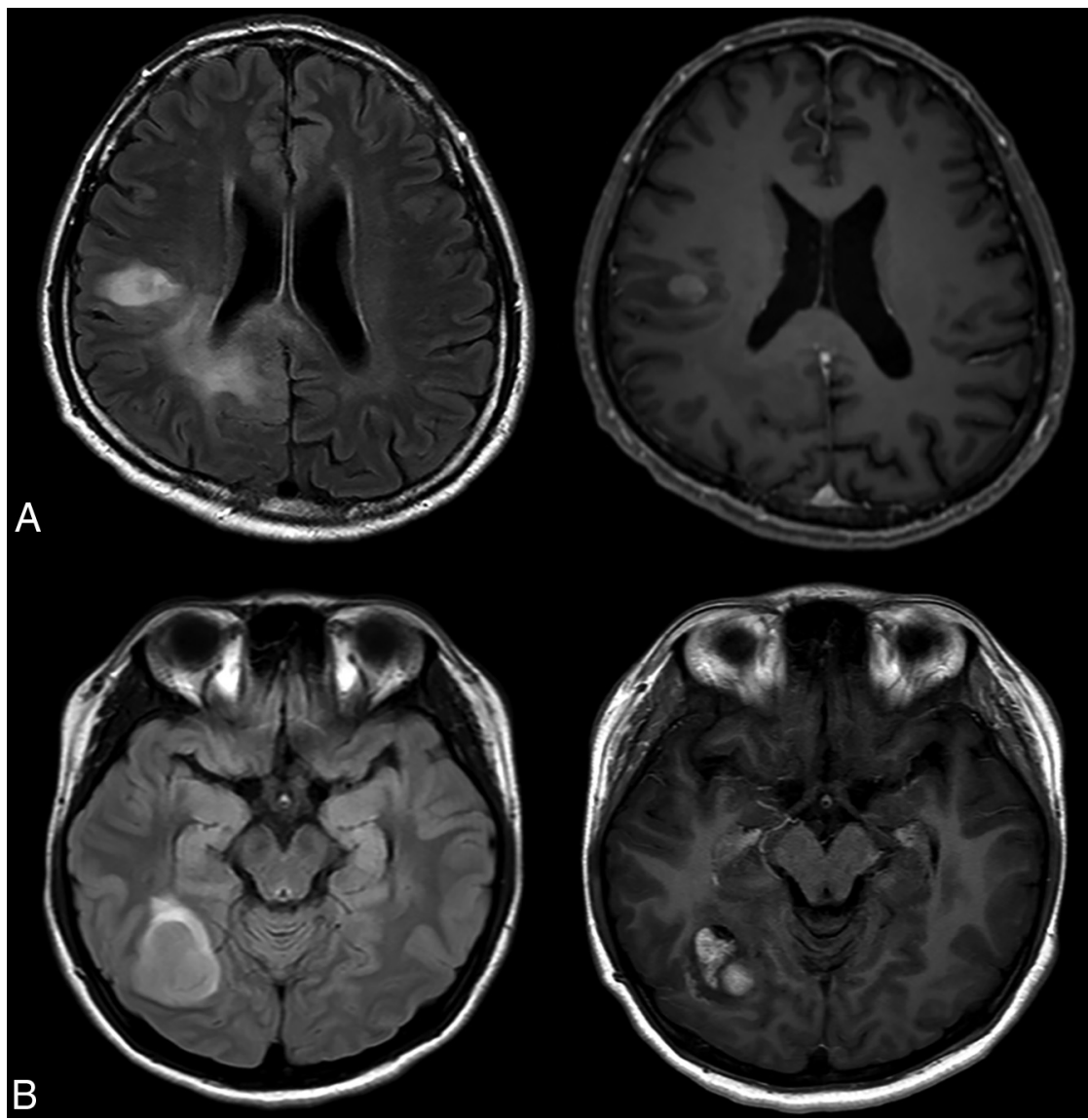

FIG 2. Representative cases of $I D H$ wild-type lower-grade gliomas with $(A)$ and without $(B)$ molecular features of GBM. A, Initial MR imaging of a 49-year-old man with IDH wild-type lowergrade glioma with molecular features of GBM, WHO grade III. MRIs reveal infiltrative T2-hyperintense tumor in the right frontal and parietal lobes extending into the corpus callosum with cortical involvement (left). Focal contrast enhancement is noted in the right frontal lobe (right). $B$, Initial MRIs of a 26-year-old woman without molecular features of GBM, WHO grade III. MRIs reveal a relatively well-defined enhancing mass with peritumoral edema in the right temporooccipital lobe without cortical involvement.
Representative figures of $I D H$ wild-type diffuse gliomas with and without molecular features of GBM are presented in Fig 2.

\section{Model 1 (Clinical Features Only)}

The predictive performances of model 1 were 0.863 ( $95 \%$ confidence interval, $0.753-0.972)$ and $0.514 \quad(95 \% \mathrm{CI}$, 0.356-0.672) in the training and the test sets, respectively.

\section{Model 2 (VASARI Features Only)}

The predictive performances of model 2 with only VASARI features were 0.988 (95\% CI, 0.969-1) and 0.648 (95\% CI, 0.511-0.784) in the training and the test sets, respectively.

\section{Model 3 (VASARI + Radiomic Features)}

In the training set, model 3 accurately predicted the status of molecular features of GBM in IDH wild-type lowergrade gliomas with high AUCs, ranging from 0.872 to 1 . In the test set, all the AUCs of models 3 with different combinations of feature-selection methods and classifiers are presented in Fig 3. Model 3 predicted the status of molecular GBM with AUCs ranging from 0.567 to 0.854 . The combination of SVM and RFE showed the highest predictive performance with an AUC of 0.854 (95\% CI, 0.766-0.941), with a sensitivity and specificity of $71.9 \%$ and $88.0 \%$, respectively.
Interobserver agreement for VASARI features was good-toexcellent (range, 0.774-1.000) (Online Supplemental Data). The imaging features with the highest interobserver agreement were tumor location, side of lesion center, presence of enhancement, and multifocality $(\kappa=1)$; the lowest interobserver agreement was found in the proportion of necrosis $(\kappa=0.774)$.

The differences of VASARI features between the IDH wildtype lower-grade gliomas with and without molecular features of GBM in the training and the test sets are provided in the Online Supplemental Data. In both the training and test sets, cortical involvement was the only feature that was significantly different between the 2 groups.

In the training set, a total of 5, 174, and 9 features were selected through LASSO, RFE, and RFE + LASSO, respectively. Five consistently selected features among all 3 methods were 2 texture features, 1 first-order feature, and 2 VASARI features: short run emphasis (T2), gray-level nonuniformity normalized (postcontrast T1), minimum (T2), infiltrative tumor growth, and cortical involvement.

\section{Model 4 (Clinical + VASARI + Radiomic Features)}

In the training set, model 4 accurately predicted the status of molecular features of GBM in IDH wild-type lower-grade gliomas with high AUCs, ranging from 0.943 to 1 . In the test set, model 4 predicted the status of molecular GBMs with AUCs ranging from 0.524 to 0.863 . The combination of SVM and RFE provided the highest predictive performance with an AUC of 0.863 (95\% CI, $0.778-0.947)$, with a sensitivity and specificity of $81.3 \%$ and $88.0 \%$, respectively.

\section{Model Comparisons}

The best-performing models in the test set from models 3 and 4 were compared with each other and with models 1 and 2 (Table 2 and Fig 4). Model 3 (AUC $=0.854)$ and model $4(\mathrm{AUC}=0.863)$ yielded significantly superior performances for molecular GBMstatus prediction compared with model 1 (AUC $=0.514 ; P<$ .001 , both) and model 2 (AUC $=0.648 ; P=.023$ and .02 , respectively). There was no significant difference between models 3 and $4(P=.476)$. 


\section{DISCUSSION}

In this study, we integrated MR imaging features with machine learning techniques to establish accurate models to identify the specific subset of $I D H$ wild-type lower-grade gliomas that had molecular features of GBM. Our models were subsequently tested using an independent test set, which proved their generalizability and robustness $(\mathrm{AUC}=0.854)$.

Several studies have reported that EGFR amplification and TERT promotor mutations are significantly associated with aggressive tumor behavior and worse prognosis in patients with lower-grade gliomas ${ }^{35-38}$ and GBMs. ${ }^{39-41}$ Particularly, because the alterations in the EGFR gene are the potential therapeutic targets, several previous studies attempted to capture the imaging signature of EGFR mutations and to detect them noninvasively in an in vivo setting using complex multiparametric MR imaging or perfusion MR imaging in patients with GBM. ${ }^{42,43}$ Also in $I D H$ wild-type lower-grade gliomas, tumors having EGFR amplification or TERT promotor mutations can be classified into "molecularly" high-grade tumors with a significantly shorter survival rate than gliomas with no mutations. ${ }^{7}$ Subsequently, the recent cIMPACT-NOW defined $I D H$ wild-type lower-grade gliomas with 1 of 3 characteristics (EGFR amplification, +7/-10 loss, or TERT promotor mutations) as "diffuse astrocytic glioma, $I D H$-wildtype, with molecular features of GBM, WHO grade IV."10
Model 3

VASARI + Radiomic features

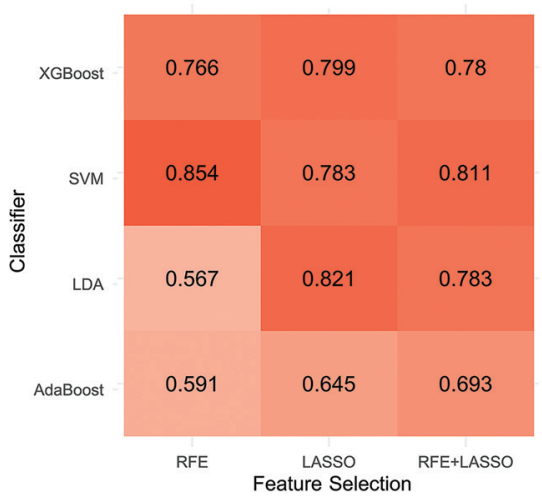

Model 4

Clinical + VASARI + Radiomic features

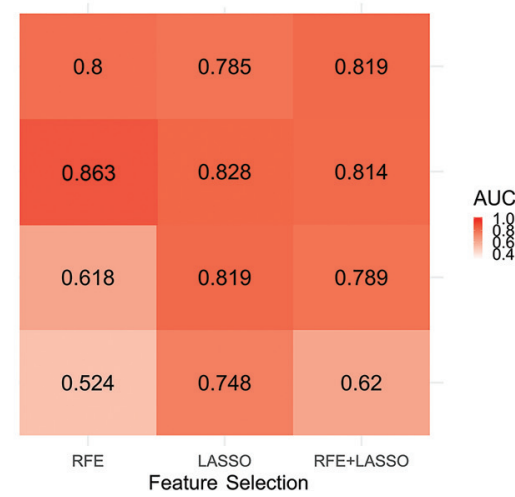

FIG 3. Heat maps illustrating the predictive performance (AUCs) of the different combinations of feature selection methods (rows) and classifiers (columns) from models 3 and 4 in the test set. LDA indicates linear discriminant analysis; AdaBoost, adaptive boosting.
Therefore, in our study, we investigated whether the subset of $I D H$ wild-type lower-grade gliomas with molecular features of GBM could be predicted noninvasively using comprehensive MR imaging analysis. Rather than considering individual EGFR amplification or TERT promotor mutation, we focused on predicting the molecular features of GBM. This focus was because the molecular features of GBM incorporate both of these genetic mutations, which enable the identification of more patients with unfavorable prognoses who might need more aggressive treatment. In addition, we included only the $I D H$ wild-type subgroup of lower-grade gliomas in our study because predicting the molecular GBM status in this subgroup might be more clinically relevant according to the cIMPACT-NOW recommendations.

The patients' age and WHO grades served as our clinical parameters, according to previous studies that reported that $I D H$ wild-type grade II and III gliomas with genetic alterations characteristic of GBM were diagnosed at a significantly older age. ${ }^{35,38}$ WHO grade III, together with high-risk genetic alterations, was also a significant prognostic factor in patients with $I D H$ wild-type lower-grade gliomas. ${ }^{7,38}$ However, our model with only clinical features showed a poor predictive performance $(\mathrm{AUC}=0.514)$. Furthermore, comprehensive MR imaging analysis using VASARI allowed slightly better prediction for the molecular GBM status than the clinical features; however, it still showed unsatisfactory performance $(\mathrm{AUC}=0.648)$. A recent study revealed that radiomics allowed the prediction of EGFR expression in patients with diffuse lower-grade gliomas. ${ }^{17}$ Our study results proved that radiomics functions equally well in the $I D H$ wild-type subgroup of lowergrade gliomas because it can accurately stratify patients according to the molecular GBM status when added to the VASARI features $(A U C=0.854)$. It is important to identify the molecular features of GBM beyond $I D H$ mutation status noninvasively because they convey prognostic information that could help clinicians decide different treatment schemes for their patients.

Among 5 consistently selected features, 2 features were texture-based. Texture analysis refers to a variety of

Table 2: Highest predictive performances of different models in identifying the molecular features of glioblastomas in IDH wildtype lower-grade gliomas in the test set

\begin{tabular}{|c|c|c|c|c|c|}
\hline Model & $\begin{array}{c}\text { Feature Selection + } \\
\text { Classifier }\end{array}$ & AUC & \multicolumn{3}{|c|}{$P$ Values for Model Comparisons } \\
\hline Model 1 (clinical features) & NA & $0.514(0.356-0.672)$ & & & \\
\hline Model 2 (VASARI features) & NA & $0.648(0.511-0.784)$ & & & \\
\hline Model 3 (VASARI + radiomics features) & RFE + SVM & $0.854(0.766-0.941)$ & $<.001^{\mathrm{a}}$ & $.023^{\mathrm{b}}$ & \\
\hline Model 4 (clinical + VASARI + radiomics features) & RFE + SVM & $0.863(0.778-0.947)$ & $<.001^{\mathrm{a}}$ & $.02^{\mathrm{b}}$ & $.476^{\mathrm{c}}$ \\
\hline
\end{tabular}

Note:-NA indicates not applicable.

${ }^{a}$ Compared with model 1.

${ }^{\mathrm{b}}$ Compared with model 2.

${ }^{\mathrm{c}}$ Compared with model 3. 


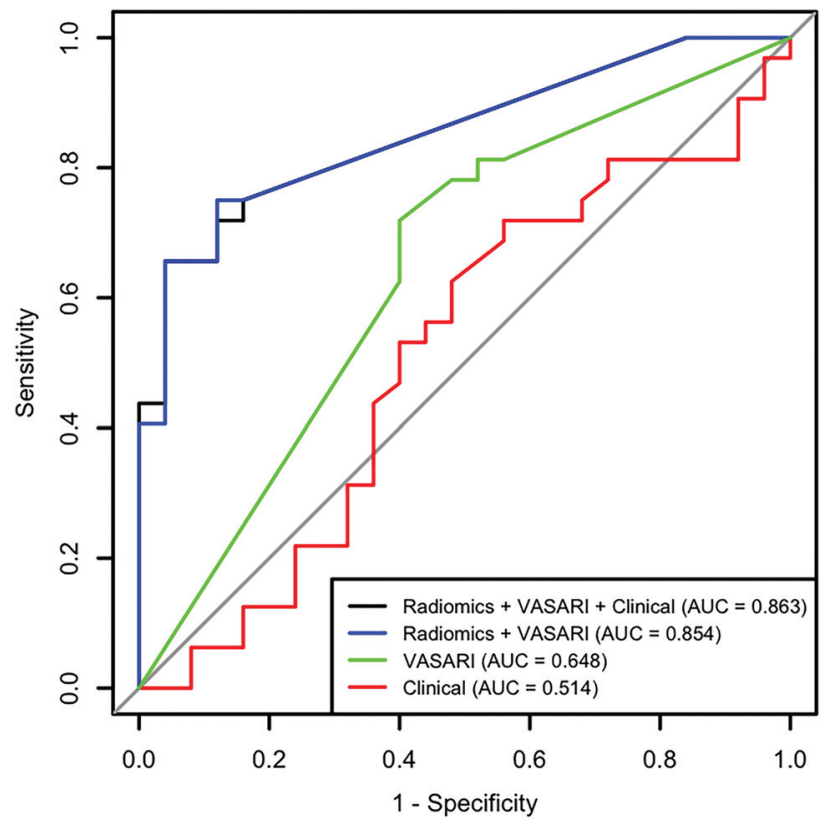

FIG 4. Receiver operating characteristic curves of models $1,2,3$, and 4 with the highest predictive performance. The combination of SVM and RFE shows the highest predictive performance in both models 3 and 4.

mathematic methods used to quantify the gray-level spatial variations within an image to derive textural features, which reflect intratumoral heterogeneity. ${ }^{16}$ These textural features reflecting intratumoral heterogeneity have shown potential in predicting specific genetic mutations ${ }^{17-19}$ and survival rates ${ }^{44}$ in patients with lower-grade gliomas. Specifically, one of the gray-level run length matrix features called short run emphasis derived from T2WI was significantly associated with EGFR expression in patients with lower-grade gliomas in a recent study, ${ }^{17}$ which was also selected as a potential predictor in our study. Another selected gray-level run length matrix feature called gray-level nonuniformity normalized derived from postcontrast $\mathrm{T} 1$ has also been reported to have a significant association with TERT promotor mutation status in lower-grade gliomas. ${ }^{45}$ Because the gray-level run length matrix is a measurement of regional heterogeneity, ${ }^{16}$ tumors with higher values of those features are more likely to carry molecular features of GBM.

The proportions of tumors with molecular features of GBM were different across the 3 datasets. In the TCGA dataset, tumors with molecular features of GBM were approximately $80 \%$, which was the highest. In the test set, approximately half of the tumors had molecular features of GBM. We believe that these differences reflect the differences in patient characteristics at each institution. The innate heterogeneity within the TCGA dataset might have also contributed to the differences. However, MR imaging features showed superior predictive performances when tested in the new external validation set, regardless of the difference in proportions of the tumors with features of molecular GBM, which proved their robustness.

There are several limitations of this study. First, this was a retrospective study with a small number of patients available from each dataset. Information on EGFR amplification or TERT promotor mutation was not available in many cases, and only a small number of tumors with known genetic alterations were studied. It would be highly desirable if we could compare the performances of different combinations of feature-selection methods and classifiers on a separate validation set and then test the final model on a test set, to obtain more reliable results. However, because the number of enrolled patients was low, we were not able to have another separate validation set. Instead, we performed 5-fold cross-validation with 3 repetitions in the training set. Future studies with larger numbers of patients are required to validate our study results.

Second, the combination of whole chromosome 7 gain and 10 loss is also one of the key genetic alterations that determine the status of molecular GBM; however, this could not be evaluated because the relevant information was not available in the test set. Further studies using a larger number of patients with available $+7 /-10$ information are required to validate our study results. Third, although there have been some issues of interobserver variability in the grading of gliomas, ${ }^{46-48}$ we could not calculate the interrater reliability of the WHO grade in this study because 1 senior neuropathologist reviewed the pathologic reports of enrolled subjects. In addition, the molecular subtyping of gliomas was performed by senior pathologists in each academic institution according to 2016 WHO classification; however, whether there were any discrepancies between the pathologists was not evaluated. Fourth, we did not perform the skull-stripping before the signal intensity normalization in the preprocessing for the radiomics feature extraction. However, because all patients' MR images were processed without skull-stripping uniformly, the final results of our study might not have been affected profoundly. Furthermore, we believe that it is noteworthy to investigate the predictive potential of radiomics in future studies with various research topics: whether radiomics could accurately classify $I D H$ wild-type lower-grade gliomas with molecular features of GBM and grade IV GBM or could predict the WHO grade in gliomas with specific genetic mutations such as EGFR amplification or TERT promotor mutation.

\section{CONCLUSIONS}

MR imaging features combined with machine learning classifiers can noninvasively predict the molecular features of GBM in $I D H$ wild-type lower-grade gliomas with high accuracy.

Disclosures: Sung Soo Ahn-RELATED: Grant: Basic Science Research Program through the National Research Foundation of Korea, Comments: 2017RIDIA1B03030440, 2020RIA2C1003886.* *Money paid to the institution.

\section{REFERENCES}

1. Yan H, Parsons DW, Jin G, et al. IDH1 and IDH2 mutations in gliomas. N Engl J Med 2009;360:765-73 CrossRef Medline

2. Louis DN, Perry A, Reifenberger G, et al. The 2016 World Health Organization Classification of Tumors of the Central Nervous System: a summary. Acta Neuropathol 2016;131:803-20 CrossRef Medline

3. Jiao Y, Killela PJ, Reitman ZJ, et al. Frequent ATRX, CIC, FUBP1 and IDH1 mutations refine the classification of malignant gliomas. Oncotarget 2012;3:709-22 CrossRef Medline

4. Brat DJ, Verhaak RG, Aldape KD, et al; Cancer Genome Atlas Research Network. Comprehensive, integrative genomic analysis of 
diffuse lower-grade gliomas. $N$ Engl J Med 2015;372:2481-98 CrossRef Medline

5. Metellus P, Coulibaly B, Colin C, et al. Absence of $I D H$ mutation identifies a novel radiologic and molecular subtype of WHO grade II gliomas with dismal prognosis. Acta Neuropathol 2010;120:71929 CrossRef Medline

6. Eckel-Passow JE, Lachance DH, Molinaro AM, et al. Glioma groups based on $1 \mathrm{p} / 19 \mathrm{q}, I D H$, and TERT promoter mutations in tumors. N Engl J Med 2015;372:2499-508 CrossRef Medline

7. Aibaidula A, Chan AK, Shi Z, et al. Adult IDH wild-type lowergrade gliomas should be further stratified. Neuro Oncol 2017; 19:1327-37 CrossRef Medline

8. Chan AK, Yao Y, Zhang Z, et al. TERT promoter mutations contribute to subset prognostication of lower-grade gliomas. Mod Pathol 2015;28:177-86 CrossRef Medline

9. Chan AK, Yao Y, Zhang Z, et al. Combination genetic signature stratifies lower-grade gliomas better than histological grade. Oncotarget 2015;6:20885-901 CrossRef Medline

10. Brat DJ, Aldape K, Colman H, et al. cIMPACT-NOW update 3: recommended diagnostic criteria for "Diffuse astrocytic glioma, IDHwildtype, with molecular features of glioblastoma, WHO grade IV." Acta Neuropathol 2018;136:805-10 CrossRef Medline

11. Gutman DA, Cooper LA, Hwang SN, et al. MR imaging predictors of molecular profile and survival: multi-institutional study of the TCGA glioblastoma data set. Radiology 2013;267:560-69 CrossRef Medline

12. Gutman DA, Dunn WD, Grossmann P, et al. Somatic mutations associated with MRI-derived volumetric features in glioblastoma. Neuroradiology 2015;57:1227-37 CrossRef Medline

13. Park YW, Han K, Ahn SS, et al. Prediction of IDH1-mutation and 1p/19q-codeletion status using preoperative MR imaging phenotypes in lower grade gliomas. AJNR Am J Neuroradiol 2018;39:3742 CrossRef Medline

14. Gillies RJ, Kinahan PE, Hricak H. Radiomics: images are more than pictures, they are data. Radiology 2016;278:563-77 CrossRef Medline

15. Aerts HJ, Velazquez ER, Leijenaar RT, et al. Decoding tumour phenotype by noninvasive imaging using a quantitative radiomics approach. Nat Commun 2014;5:4006 CrossRef Medline

16. Molina D, Pérez-Beteta J, Martínez-González A, et al. Influence of gray level and space discretization on brain tumor heterogeneity measures obtained from magnetic resonance images. Comput Biol Med 2016;78:49-57 CrossRef Medline

17. Li Y, Liu X, Xu K, et al. MRI features can predict EGFR expression in lower grade gliomas: a voxel-based radiomic analysis. Eur Radiol 2018;28:356-62 CrossRef Medline

18. Li Y, Qian Z, Xu K, et al. MRI features predict p53 status in lowergrade gliomas via a machine-learning approach. Neuroimage Clin 2018;17:306-11 CrossRef Medline

19. Li Y, Qian Z, Xu K, et al. Radiomic features predict Ki-67 expression level and survival in lower grade gliomas. J Neurooncol 2017;135:317-24 CrossRef Medline

20. Pedano N, Flanders A, Scarpace L, et al. Radiology data from the Cancer Genome Atlas low grade glioma [TCGA-LGG] collection. The Cancer Imaging Archive 2016 CrossRef

21. Wiki for the VASARI feature set. The National Cancer Institute Website. https://wiki.cancerimagingarchive.net/display/Public/VASARI +Research+Project. Updated March 30, 2020. Accessed September 17, 2019

22. Tustison NJ, Avants BB, Cook PA, et al. N4ITK: improved N3 bias correction. IEEE Trans Med Imaging 2010;29:1310-20 CrossRef Medline

23. Sled JG, Zijdenbos AP, Evans AC. A nonparametric method for automatic correction of intensity nonuniformity in MRI data. IEEE Trans Med Imaging 1998;17:87-97 CrossRef Medline

24. Shinohara RT, Sweeney EM, Goldsmith J, et al. Statistical normalization techniques for magnetic resonance imaging. Neuroimage Clin 2014;6:9-19 CrossRef Medline
25. van Griethuysen JJ, Fedorov A, Parmar C, et al. Computational radiomics system to decode the radiographic phenotype. Cancer Res 2017;77:e104-07 CrossRef Medline

26. Takano S, Tian W, Matsuda M, et al. Detection of IDH1 mutation in human gliomas: comparison of immunohistochemistry and sequencing. Brain Tumor Pathol 201 1;28:115-23 CrossRef Medline

27. Choi J, Lee EY, Shin KJ, et al. IDH1 mutation analysis in low cellularity specimen: a limitation of diagnostic accuracy and a proposal for the diagnostic procedure. Pathol Res Pract 2013;209:284-90 CrossRef Medline

28. Sahm F, Schrimpf D, Jones DTW, et al. Next-generation sequencing in routine brain tumor diagnostics enables an integrated diagnosis and identifies actionable targets. Acta Neuropathol 2016;131:903-10 CrossRef Medline

29. Na K, Kim HS, Shim HS, et al. Targeted next-generation sequencing panel (TruSight Tumor 170) in diffuse glioma: a single institutional experience of 135 cases. J Neurooncol 2019;142:445-54 CrossRef Medline

30. Friedman J, Hastie T, Tibshirani R. Regularization paths for generalized linear models via coordinate descent. J Stat Softw 2010;33:122 Medline

31. Dormann CF, Elith J, Bacher S, et al. Collinearity: a review of methods to deal with it and a simulation study evaluating their performance. Ecography 2013;36:27-46 CrossRef

32. Kuhn M. Building predictive models in $\mathbf{R}$ using the caret package. $J$ Stat Soft 2008;28 CrossRef

33. Landis JR, Koch GG. The measurement of observer agreement for categorical data. Biometrics 1977;33:159-74 Medline

34. DeLong ER, DeLong DM, Clarke-Pearson DL. Comparing the areas under two or more correlated receiver operating characteristic curves: a nonparametric approach. Biometrics 1988;44:83745 Medline

35. Bale TA, Jordan JT, Rapalino O, et al. Financially effective test algorithm to identify an aggressive, EGFR-amplified variant of $I D H$ wildtype, lower-grade diffuse glioma. Neuro Oncol 2019;21:596-605 CrossRef Medline

36. Weller M, Weber RG, Willscher E, et al. Molecular classification of diffuse cerebral WHO grade II/III gliomas using genome- and transcriptome-wide profiling improves stratification of prognostically distinct patient groups. Acta Neuropathol 2015;129:679-93 CrossRef Medline

37. Wijnenga MM, Dubbink HJ, French PJ, et al. Molecular and clinical heterogeneity of adult diffuse low-grade $I D H$ wild-type gliomas: assessment of TERT promoter mutation and chromosome 7 and 10 copy number status allows superior prognostic stratification. Acta Neuropathol 2017;134:957-59 CrossRef Medline

38. Aoki K, Nakamura H, Suzuki H, et al. Prognostic relevance of genetic alterations in diffuse lower-grade gliomas. Neuro Oncol 2018;20:66-77 CrossRef Medline

39. Stichel D, Ebrahimi A, Reuss D, et al. Distribution of EGFR amplification, combined chromosome 7 gain and chromosome 10 loss, and TERT promoter mutation in brain tumors and their potential for the reclassification of IDH wild type astrocytoma to glioblastoma. Acta Neuropathol 2018;136:793-803 CrossRef Medline

40. Gupta A, Young RJ, Shah AD, et al. Pretreatment dynamic susceptibility contrast MRI perfusion in glioblastoma: prediction of EGFR gene amplification. Clin Neuroradiol 2015;25:143-50 CrossRef Medline

41. Ivanidze J, Lum M, Pisapia D, et al. MRI features associated with TERT promoter mutation status in glioblastoma. J Neuroimaging 2019;29:357-63 CrossRef Medline

42. Akbari $\mathrm{H}$, Bakas S, Pisapia JM, et al. In vivo evaluation of EGFRvIII mutation in primary glioblastoma patients via complex multiparametric MRI signature. Neuro Oncol 2018;20:1068-79 CrossRef Medline

43. Bakas $\mathrm{S}$, Akbari $\mathrm{H}$, Pisapia J, et al. In vivo detection of EGFRvIII in glioblastoma via perfusion magnetic resonance imaging signature 
consistent with deep peritumoral infiltration: the $\varphi$-Index. Clin Cancer Res 2017;23:4724-34 CrossRef Medline

44. Liu X, Li Y, Qian Z, et al. A radiomic signature as a non-invasive predictor of progression-free survival in patients with lowergrade gliomas. Neuroimage Clin 2018;20:1070-77 CrossRef Medline

45. Jiang C, Kong Z, Zhang Y, et al. Conventional magnetic resonance imaging-based radiomic signature predicts telomerase reverse transcriptase promoter mutation status in grade II and III gliomas. Neuroradiology 2020;62:803-13 CrossRef Medline
46. Kros JM. Grading of gliomas: the road from eminence to evidence. J Neuropathol Exp Neurol 2011;70:101-09 CrossRef Medline

47. Prayson RA, Agamanolis DP, Cohen ML, et al. Interobserver reproducibility among neuropathologists and surgical pathologists in fibrillary astrocytoma grading. J Neurol Sci 2000;175:3339 CrossRef Medline

48. Vigneswaran K, Neill S, Hadjipanayis CG. Beyond the World Health Organization grading of infiltrating gliomas: advances in the molecular genetics of glioma classification. Ann Transl Med 2015;3:95 CrossRef Medline 